\title{
Process for the Preparation of Chromones, Isoflavones and Homoisoflavones Using Vilsmeier Reagent Generated from Phthaloyl Dichloride and DMF
}

\author{
Santosh Kumar Yadav \\ Department of Organic Chemistry \& FDW, Andhra University, Visakhapatnam, India \\ Email: skgoityadav@gmail.com \\ Received 6 September 2014; revised 21 October 2014; accepted 6 November 2014 \\ Copyright (C) 2014 by author and Scientific Research Publishing Inc. \\ This work is licensed under the Creative Commons Attribution International License (CC BY). \\ http://creativecommons.org/licenses/by/4.0/ \\ (c) (i) Open Access
}

\begin{abstract}
Vilsmeier reagent formed from phthaloyl dichloride and DMF was found to be very effective for converting 2-hydroxyacetophenones, deoxybenzoins and dihydrochalcones into corresponding chromones, isoflavones and homoisoflavones with excellent yield. This method offers significant advantages such as efficiency and mild reaction conditions with shorter reaction time.
\end{abstract}

\section{Keywords}

Phthaloyl Dichloride, Dimethylformamide, Chromones, Isoflavones, Homoisoflavones, $\mathrm{BF}_{3} \cdot \mathrm{Et}_{2} \mathrm{O}$, Vilsmeier Reagent

\section{Introduction}

In recent years, scientific interest towards chromones (2), isoflavones (9) and homoisoflavones (10) has increased. It is due to the limited distribution of these compounds in the plant kingdom and the possible health effect these compounds exhibit. The development of new methodologies for the synthesis of these compounds is important. It is known that certain natural and synthetic chromone derivatives possess important biological activities such as antitumor [1], antihepatotonic, antioxidant [2], anti-inflammatory [3], antispasmolytic, estrogenic [4] and antibacterial activities [5]. Isoflavones are a privileged class of natural products which are produced by plants mainly in the species of Leguminosae family to protect themselves from environmental stress and are present in dietary components such as fruits, cabbage, soybeans, grains, hops and redwines. Isoflavones possess many biological activities such as estrogenic [6], anticancer [7], antibacterial [8], antimicrobial [9], antiulcer [10] 
and protein tyrosine kinase inhibitor [11]. Search for new methodologies for the synthesis of isoflavones continues to be of great interest for organic chemists. The two most popular pathways for the synthesis of isoflavones are the deoxybenzoin and the chalcone routes. In the first route, isoflavones are synthesized by the ring closure of deoxybenzoin with $\mathrm{C}_{1}$ unit by using different reagents such as ethoxalylchoride in pyridine [12], triethylorthoformate with pyridine and piperidine [13], N, N-dimethylformamide and $\mathrm{BF}_{3} \cdot \mathrm{Et}_{2} \mathrm{O}$ with $\mathrm{MeSO}_{2} \mathrm{Cl}$ [14] [15] or $\mathrm{POCl}_{3}$ [16], anhydrous ethyl formate and powdered sodium [17], acetic anhydride and sodium acetate [18] [19], acetic-formic anhydride [20], N-formylimidazole in anhydrous THF [21] and phenyliodine (III)bis (trifluoroacetate) [22] [23]. In the second route, isoflavones are synthesized by oxidative rearrangement of a chalcone using reagents like thallium(III)nitrate [24]-[29] and thallium(III)acetate [30]-[32]. Similarly, rearrangement of chalcone epoxide with $\mathrm{BF}_{3} \cdot \mathrm{Et}_{2} \mathrm{O}$ is followed by catalytic hydrogenation [33] [34]. Other routes include the conversion of flavanones into isoflavones by thallium(III)nitrate in a mixture of $\mathrm{CH}_{3} \mathrm{OH}$ and $\mathrm{CHCl}_{3}$ [35], arylation of 4-chromanones with 4, 5-dimethoxy-o-benzoquinone in anhydrous DMSO followed by acidification [36] [37], tetrakis (triphenylphosphine) palladium (0) catalyzed cross-coupling reactions of 3-iodochromone with arylboronic acid [38], etc. However, the reported syntheses of many isoflavones including daidzein and formononetin are time-consuming (Bass, 1976 [44], Baker et al., 1953 [12], Farkas et al., 1971 [43], Pelter and Foot, 1976 [50], Yoder et al., 1954 [51]).

Homoisoflavonoids are a class of naturally occurring oxygen containing heterocyclic compounds. Both natural and synthetic homoisoflavonoids exhibit numerous biological activities [39]-[41] like antifungal, hypocholesterolemic, antimutagenic, antirhinovirus, antiallergic, angio productive activity, antihistaminic activity, antiinflammatory, antioxidant, antiviral, cough relief, inhibition of platelet aggregation etc. Homoisoflavonoids can be synthesized either by the condensation of 4-chromanones with arylaldehdes in methanol by passing $\mathrm{HCl}$ gas or by using piperidine as a base followed by isomerisation of the double bond using $\mathrm{Pd} / \mathrm{C}$ at $250^{\circ} \mathrm{C}$ [42] or by the extension of one carbon in dihydrochalcone using ethylformate/sodium [43] or $\mathrm{BF}_{3} \cdot \mathrm{Et}_{2} \mathrm{O}$ and DMF with $\mathrm{MeSO}_{2} \mathrm{Cl}$ [44] or $\mathrm{PCl}_{5}$ [45] etc.Both the methods have disadvantages; while the first method has multiple steps, in the second method, the phenolic hydroxyls have to be protected to get chalcones in good yield.

However, most of the methods reported for the synthesis of chromones, isoflavones and homoisoflavones suffer from harsh reaction conditions, poor substituent tolerance, long reaction times, and low to moderate yields. Therefore, developing a milder and more general procedure for chromones, isoflavones, and homoisoflavones is still highly desirable. It was reported that when DMF was treated with phthaloyl dichloride in 1,4-dioxane at $40^{\circ} \mathrm{C}$ for $3 \mathrm{~h}$ precipitated only vilsmeier reagent as a solid form while the co-product phthalic anhydride was dissolved in a solvent [46]. The precipitates were collected by filtration through a glass-filter funnel under nitrogen atmosphere. The residue was dried in vacuo to give white crystals, which was identified as the vilsmeier reagent by comparison with authentic supplied by Aldrich Chemical Co. Vilsmeier reagent is well known as a versatile synthetic tool for the formylation of electron-rich aromatics, chlorination of alcohols, conversion of carboxylic acid into the corresponding acid chloride and so on [47] [48]. A series of 2-hydroxyacetophenone, deoxybenzoin and dihydrochalcone was cyclized with a one carbon unit by using this reagent. The reaction requires a short reaction time, mild reaction conditions and easy work-up. Products obtained by this methodology do not have contaminants such as sulphur or phosphine obtained through DMF with $\mathrm{MeSO}_{2} \mathrm{Cl}$ [44] or $\mathrm{PCl}_{5}$ [45]. Naturally, occurring isoflavones such as formononetin (9c), daidzein (9d) and retusin (9h) was synthesized by applying this methodology. To the best of researcher knowledge, the synthesis of chromones, isoflavones and homoisoflavones using vilsmeier reagent formed from phthaloyl dichloride and DMF has not been reported.

\section{Results and Discussion}

The method first involved the preparation of vilsmeier reagent, for this, to a mixture of DMF in 1,4-dioxane was added phthaloyl dichloride at room temperature, and then the whole mixture was stirred at $40^{\circ} \mathrm{C}$ for $3 \mathrm{~h}$ (Scheme 1). The white precipitates of (chloromethylene) dimethyliminiumchloride (VR) were isolated by filtration under a nitrogen atmosphere.

First application came with its usage in preparation of chromones in which 1 equiv. of substituted 2-hydroxyacetophenone was dissolved in 3 equiv. of $\mathrm{BF}_{3} \cdot \mathrm{Et}_{2} \mathrm{O}$ and $\mathrm{DMF}$ was added drop wise with stirring at $10^{\circ} \mathrm{C}$. Then whole reaction mixture was transferred slowly with continuous stirring into 1 equiv. of vilsmeier reagent. The reaction mixture was stirred at $50^{\circ} \mathrm{C}$ for 30 minutes (Scheme 2). The completion of reaction was monitored by TLC. The reaction mixture was poured into $3 \mathrm{~N} \mathrm{HCl}$, extracted with EtOAc, dried over $\mathrm{Na}_{2} \mathrm{SO}_{4}$ and concen- 
<smiles>CN(C)C(=O)[O-]</smiles>

Scheme 1. Preparation of vilsmeier reagent from phthaloyl dichloride and DMF.<smiles>[R]c1cc([R3])c(C(C)=O)c(O)c1[R]</smiles>

1

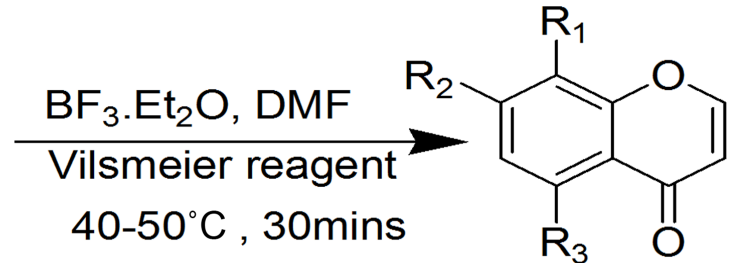

2

\section{Scheme 2. Synthesis of chromones.}

trated. The pure compound was then harvested with column chromatography.

The next success came with its usage in the preparation of isoflavones and homoisoflavones. For this, deoxybenzoins (7) and dihydrochalcones (8) were prepared by the published procedure [49] from phenyl acetic acid and 3-phenylpropanoic acid respectively with substituted phenols by Friedel-Crafts acylation using $\mathrm{BF}_{3} \cdot \mathrm{Et}_{2} \mathrm{O}$ which served as the Lewis acid for the acylation as well as the solvent for the reaction. The acylation was carried out at $85^{\circ} \mathrm{C}-90^{\circ} \mathrm{C}$. The completion of reaction was monitored by TLC. In most cases, the reaction was completed within 90 minutes. However, the substitution pattern as well as the presence of unprotected hydroxyl groups on the aromatic rings influenced the reaction time and the product yield. Conversion of these intermediate deoxybenzoin and dihydrochalcone into their respective isoflavones and homoisoflavones can be carried out either directly by treating with vilsmeier reagent, a minimum of 5 equivalents of $\mathrm{BF}_{3} \cdot \mathrm{Et}_{2} \mathrm{O}$ was required (Method A) or these intermediates were isolated, purified and then cyclised with vilsmeier reagent, for this a minimum of 3 equivalents of $\mathrm{BF}_{3} \cdot \mathrm{Et}_{2} \mathrm{O}$ was required (Method $\mathrm{B}$ ) (Scheme 3). In all cases, the reaction was completed in 30-40 mins and the products were characterized by their spectral data (IR, NMR, and mass spectrometry).

To explain the formation of chromones (2), isoflavones (9), and homoisoflavones (10), a suggested mechanism is shown in Figure 1. The mechanism involves the addition of a vilsmeier reagent (I) to the acetophenone. $\mathrm{BF}_{3}$ complex (II) to form (III), with subsequent nucleophilic attack of the hydroxyl group of 2-hydroxyacetophenone to form (IV), which is deaminated to form the final product (V).

\section{Conclusion}

A variety of chromones, isoflavones and homoisoflavones were synthesized in excellent yields using vilsmeier reagent generated from phthaloyl dichloride and DMF as the key reagent. The ready availability, low cost of phthaloyl dichloride and DMF, high activity of isolated vilsmeier reagent, the short reaction time, the mild reaction conditions, and the easy purification of the products make this an attractive new method for the synthesis of chromones, isoflavones and homoisoflavones, etc.

\section{Experimental}

\subsection{General Remarks}

All synthesized compound melting points were recorded on a Mel-Temp melting point apparatus in open capillaries and are uncorrected. Reactions requiring anhydrous conditions were performed in flame-dried glassware, and cooled under an argon or nitrogen atmosphere. Acme silica gel G and silica gel (100 - 200 mesh) were used for analytical thin-layer chromatography and column chromatography. Visualization of the resulting chroma- 


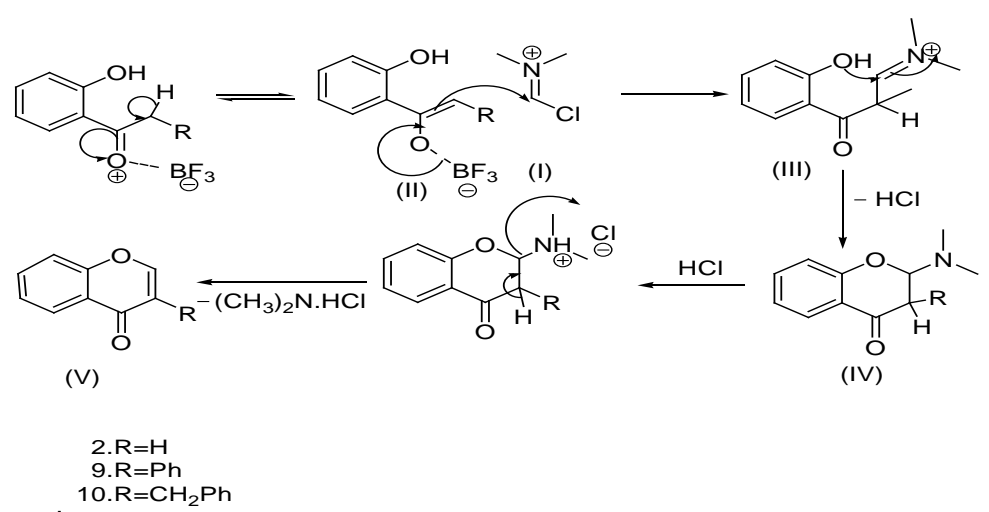

Figure 1. Plausible mechanism for the formation of 2, 9, and 10.

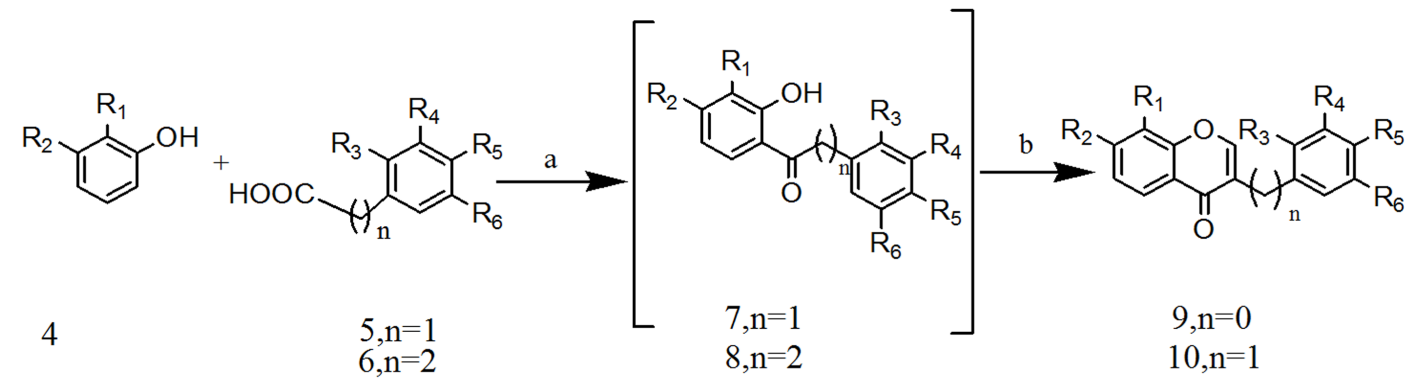

Reagents and conditions:(a) $\mathrm{BF}_{3} \cdot \mathrm{Et}_{2} \mathrm{O}, 90^{\circ} \mathrm{C}, 90 \mathrm{~min},(\mathrm{~b}) \mathrm{DMF}, 10^{\circ} \mathrm{C}$, Vilsmeier reagent, $60^{\circ} \mathrm{C}, 30-40 \mathrm{~min}$

Scheme 3. Synthesis of isoflavones (9a-9h) and homoisoflavones (10a-10f).

tograms was done by looking under an ultraviolet lamp ( $\lambda=254 \mathrm{~nm}$ ). IR spectra were recorded on a Perkin-Elmer BX1 FTIR spectrophotometer and ${ }^{1} \mathrm{H}$ NMR (400 MHz) and ${ }^{13} \mathrm{C}$ NMR (100 MHz) spectra were recorded on a Bruker AMX $400 \mathrm{MHz}$. NMR spectrometer using TMS as the internal standard and the values for chemical shifts (_) being given in parts per million and coupling constants $(J)$ in hertz. Mass spectra were recorded on an Agilent 1100 LC/MSD.

\subsection{Preparation of Vilsmeier Reagent from Phthaloyl Dichloride and DMF in 1,4-Dioxane}

A mixture of DMF $30 \mathrm{~g}(0.41 \mathrm{~mol})$ and phthaloyl dichloride $90 \mathrm{~g}(0.44 \mathrm{~mol})$ in 1,4-dioxane $(330 \mathrm{~mL})$ was stirred at $40^{\circ} \mathrm{C}$ for $3 \mathrm{~h}$. The white precipitates of (chloromethylene)dimethyliminium chloride (VR) that formed were collected by filtration under a nitrogen atmosphere, washed with 1,4-dioxane $(100 \mathrm{~mL} \times 2)$ and hexane (100 mL), and dried under reduced pressure, $41 \mathrm{gm}$ (78\% yield).

\subsection{Preparation of Chromones (2a-2d)}

DMF (4.6 mL) was added to a stirred solution of 2-hydroxyacetophenone (3 mmol) in $\mathrm{BF}_{3} \cdot \mathrm{Et}_{2} \mathrm{O}(7.5 \mathrm{mmol})$ at $10^{\circ} \mathrm{C}$ for $5 \mathrm{~min}$. The reaction mixture was then added to the vilsmeier reagent ( $\left.4.5 \mathrm{mmol}\right)$ drop wise with stirring at room temperature. After completion of addition, the reaction mixture was stirred at $50^{\circ} \mathrm{C}$ for $30-40$ mins and poured into boiling dilute $\mathrm{HCl}$ slowly and cooled. The solution was extracted with ethyl acetate $(30 \mathrm{~mL} \times 2)$ and the combined organic layer was dried over anhydrous $\mathrm{Na}_{2} \mathrm{SO}_{4}$. The crude obtained after evaporation of the solvent was chromatographed over silica gel column using chloroform-methanol mixtures as eluent to give 2a-2d.

\subsection{Chromen-4-One (2a) (Table 1, Entry 1)}

Colorless solid; yield $376 \mathrm{mg}(80 \%)$; mp $55^{\circ} \mathrm{C}-58^{\circ} \mathrm{C} .{ }^{1} \mathrm{H}$ NMR $\left(400 \mathrm{MHz}\right.$, DMSO-d $\left.\mathrm{d}_{6}\right) \_$: 6.32 (d, J = $5.6 \mathrm{~Hz}$, 1H), 7.35 - $7.43(\mathrm{~m}, 2 \mathrm{H}), 7.64(\mathrm{t}, \mathrm{J}=7.6 \mathrm{~Hz}, 1 \mathrm{H}), 7.86(\mathrm{~d}, \mathrm{~J}=5.6 \mathrm{~Hz}, 1 \mathrm{H}), 8.18$ (d, J = 7.6 Hz, 1H). ${ }^{13} \mathrm{C} \mathrm{NMR}$ $(100 \mathrm{MHz}, \text { DMSO-d })_{6}=111.9,117.2,123.9,124.3,124.7,132.8,154.5,155.5,176.6$. LC-MS: $\mathrm{m} / \mathrm{z}: 147$ [M + 
Table 1. Synthesis of chromones (2a-2d).

\begin{tabular}{cccccc}
\hline S. No. & Entry & $\mathrm{R}_{1}$ & $\mathrm{R}_{2}$ & $\mathrm{R}_{3}$ & 2 Yield (\%) \\
\hline 1 & $\mathrm{a}$ & $\mathrm{H}$ & $\mathrm{HH}$ & $\mathrm{H}$ & 80 \\
2 & $\mathrm{~b}$ & $\mathrm{H}$ & $\mathrm{OH}$ & $\mathrm{H}$ & 85 \\
3 & $\mathrm{c}$ & $\mathrm{OH}$ & $\mathrm{OH}$ & $\mathrm{H}$ & 87 \\
4 & $\mathrm{~d}$ & $\mathrm{H}$ & $\mathrm{OH}$ & $\mathrm{OH}$ & 89 \\
\hline
\end{tabular}

$1]^{+}$. Anal.calcd. for $\mathrm{C}_{9} \mathrm{H}_{6} \mathrm{O}_{2}$ : C 73.97, H 4.14; Found: C 73.94, H 4.19.

\subsection{7-Hydroxy-4H-Chromen-4-One (2 b) (Table 1, Entry 2)}

Pale brown solid; yield $412 \mathrm{mg}(85 \%)$; $\mathrm{mp} 206^{\circ} \mathrm{C}-208^{\circ} \mathrm{C} .{ }^{1} \mathrm{H}$ NMR (400 MHz, DMSO-d 6 )_: $6.21(\mathrm{~d}, \mathrm{~J}=6.0 \mathrm{~Hz}$, $1 \mathrm{H}), 6.84$ (d, J = 2.4 Hz, 1H), 6.91 (dd, J = 2.4, 8.4 Hz, 1H), 7.87 (d, J = 8.4 Hz, 1H), 8.14 (d, J = 6.0 Hz, 1H), 10.76 (s, 1H). ${ }^{13} \mathrm{C}$ NMR (100 MHz, DMSO-d $)_{6}=102.3,111.9,115.0,117.0,126.6,156.0,157.7,162.5,175.5$. LC-MS: m/z: 161 [M-1]-. Anal.calcd. for $\mathrm{C}_{9} \mathrm{H}_{6} \mathrm{O}_{3}$ : C 66.67, H 3.73; found: C 66.65, H 3.75.

\subsection{7,8-Dihydroxy-4H-Chromen-4-One (2c) (Table 1, Entry 3)}

Brown solid; yield $466 \mathrm{mg}(87 \%)$; mp $205^{\circ} \mathrm{C}-208^{\circ} \mathrm{C} .{ }^{1} \mathrm{H}$ NMR (400 MHz, DMSO-d $)_{-}: 6.17$ (d, J = $6.0 \mathrm{~Hz}$, $1 \mathrm{H}), 6.93(\mathrm{~d}, \mathrm{~J}=6.8 \mathrm{~Hz}, 1 \mathrm{H}), 7.37$ (d, J = 6.8 Hz, 1H), 8.19 (d, J = 6.0 Hz, 1H), $9.40(\mathrm{~s}, 1 \mathrm{H}), 10.29(\mathrm{~s}, 1 \mathrm{H}) .{ }^{13} \mathrm{C}$ NMR (100 MHz, DMSO-d $\left.{ }_{6}\right)_{-}=111.3,114.0,115.0,117.8,132.9,146.9,150.0,155.7,176.0$. LC-MS: $\mathrm{m} / \mathrm{z}: 177$ [M-1] $]^{-}$. Anal.calcd. for $\mathrm{C}_{9} \mathrm{H}_{6} \mathrm{O}_{4}$ : C 60.68, H 3.39; found: C 60.63, H 3.43.

\subsection{5,7-Dihydroxy-4H-Chromen-4-One (2d) (Table 1, Entry 4)}

Brown solid; yield $474 \mathrm{mg}(89 \%)$; mp $268^{\circ} \mathrm{C}-270^{\circ} \mathrm{C} .{ }^{1} \mathrm{H}$ NMR (400 MHz, DMSO-d $)_{6}$ : 6.20 (d, J = $2.0 \mathrm{~Hz}$, $1 \mathrm{H}), 6.27$ (d, J = 6.0 Hz, 1H), 6.36 (d, J = 2.0 Hz, 1H), 8.17 (d, J = 6.0 Hz, 1H), 10.85 (s, $1 \mathrm{H}), 12.69(\mathrm{~s}, 1 \mathrm{H}) .{ }^{13} \mathrm{C}$ NMR (100 MHz, DMSO-d $\left.)_{6}\right)_{-}$93.9, 98.9, 104.8, 110.4, 149.8, 157.3, 157.7, 164.2, 181.2. LC-MS: m/z: 177 [M-1] $]^{-}$. Anal.calcd. for $\mathrm{C}_{9} \mathrm{H}_{6} \mathrm{O}_{4}$ : C 60.68, H 3.39; found: C 60.65, H 3.41.

\section{General Experimental Procedure for Isoflavones (9a-9h)}

\subsection{Method A}

A mixture of substituted phenol ( $3 \mathrm{mmol})$, phenylacetic acid ( $3 \mathrm{mmol})$, and $\mathrm{BF}_{3} \cdot \mathrm{Et}_{2} \mathrm{O}(15 \mathrm{mmol})$ was refluxed at $90^{\circ} \mathrm{C}$ for $90 \mathrm{~min}$ under Nitrogen atmosphere. The reaction mixture was then cooled to $10^{\circ} \mathrm{C}$ and $\mathrm{DMF}(4.6 \mathrm{~mL})$ was added drop wise. The above reaction mixture was then added drop wise with stirring into vilsmeier reagent $(4.5 \mathrm{mmol})$ at room temperature. After completion of addition, the reaction mixture was stirred at $60^{\circ} \mathrm{C}$ for 30 $40 \mathrm{~min}$ and poured into boiling dilute $\mathrm{HCl}$ slowly and cooled. The solution was extracted with ethylacetate (30 $\mathrm{mL} \times 2$ ) and the organic layer was dried over anhydrous $\mathrm{Na}_{2} \mathrm{SO}_{4}$. The crude obtained after evaporation of the solvent was chromatographed over a silica gel column using chloroform-methanol mixtures as eluent to give isoflavones (9a-9h).

\subsection{Method B}

A mixture of substituted phenol (3 mmol), phenylacetic acid (3 mmol), and $\mathrm{BF}_{3} \cdot \mathrm{Et}_{2} \mathrm{O}(9 \mathrm{mmol})$ was refluxed at $90^{\circ} \mathrm{C}$ for 90 min under Nitrogen atmosphere. The mixture was then poured into NaOAc solution (100 mL, 10\%) and allowed to stand for $4 \mathrm{hr}$ and the solution was extracted with EtOAc $(3 \times 100 \mathrm{~mL})$. The combined organic layer was washed with water $(20 \mathrm{~mL})$ and brine $(20 \mathrm{~mL})$ and dried over anhydrous $\mathrm{Na}_{2} \mathrm{SO}_{4}$. The crude obtained after evaporation of the solvent was chromatographed over a silica gel column using hexane-EtOAc mixtures as eluent to give deoxybenzoins (7f-7h). The purified materials were then used for the synthesis of isoflavones. A mixture of deoxybenzoin (3 mmol) and $\mathrm{BF}_{3} \cdot \mathrm{Et}_{2} \mathrm{O}(7.5 \mathrm{mmol})$ was cooled to $10^{\circ} \mathrm{C}$ and DMF (4.6 mL) was added drop wise. The cyclization procedure and work up are similar to method A. 


\subsection{7-Hydroxy-3-Phenyl-4H-Chromen-4-One (9a) (Table 2, Entry 1)}

White solid; yield (method A) $627 \mathrm{mg}(88 \%) ; \mathrm{mp} 210^{\circ} \mathrm{C}-213^{\circ} \mathrm{C}$. $1 \mathrm{H}$ NMR (400 MHz, DMSO-d6)_: 6.88 (d, J = $2.4 \mathrm{~Hz}, 1 \mathrm{H}), 6.96$ (dd, J = 2.4, $8.4 \mathrm{~Hz}, 1 \mathrm{H}), 7.34-7.44$ (m, 3H), 7.57 (d, J = 7.2 Hz, 2H), 7.99 (d, J = 8.8 Hz, $1 \mathrm{H}), 8.36(\mathrm{~s}, 1 \mathrm{H}), 10.80(\mathrm{~s}, 1 \mathrm{H}) .{ }^{13} \mathrm{C}$ NMR $\left(100 \mathrm{MHz}, \mathrm{DMSO}-\mathrm{d}_{6}\right)_{-}=102.1,115.2,116.6,123.5,127.2,127.6$, 128.0, 128.8, 132.1, 153.6, 157.4, 162.6, 174.3. LC-MS: m/z: 237 [M-1]-. Anal.calcd. for $\mathrm{C}_{15} \mathrm{H}_{10} \mathrm{O}_{3}: \mathrm{C}_{75.62, ~} \mathrm{H}$ 4.23; found: C $75.60, \mathrm{H} 4.27$.

\subsection{7-Hydroxy-3-(3-Methoxyphenyl)-4H-Chromen-4-One (9b) (Table 2, Entry 2)}

Pale pink solid; yield (method A) $715 \mathrm{mg}(89 \%)$; mp $215^{\circ} \mathrm{C}-217^{\circ} \mathrm{C} .{ }^{1} \mathrm{H}$ NMR (400 MHz, DMSO-d $)_{6}$ : 3.78 (s, 3H), 6.88 (s, 1H), 6.93 - $6.96(\mathrm{~m}, 2 \mathrm{H}), 7.13-7.15(\mathrm{~m}, 2 \mathrm{H}), 7.33(\mathrm{t}, \mathrm{J}=8.0 \mathrm{~Hz}, 1 \mathrm{H}), 7.98$ (d, J = 8.8 Hz, $1 \mathrm{H})$, $8.38(\mathrm{~s}, 1 \mathrm{H}), 10.79(\mathrm{~s}, 1 \mathrm{H}){ }^{13} \mathrm{C}$ NMR $\left(100 \mathrm{MHz}, \mathrm{DMSO}-\mathrm{d}_{6}\right)_{-}=55.06,102.1,113.2,114.6,115.2,116.6,121.1$, 123.3, 127.2, 129.0, 133.4, 153.8, 157.3, 159.0, 162.6, 174.3. LC-MS: m/z: 267 [M-1]-. Anal.calcd. for $\mathrm{C}_{16} \mathrm{H}_{12} \mathrm{O}_{4}$ : C 71.64, H 4.51; found: C 71.60, H 4.56.

\subsection{7-Hydroxy-3-(4-Methoxyphenyl)-4H-Chromen-4-One (9c) (Table 2, Entry 3)}

Off-white solid; yield (method A) $723 \mathrm{mg}(90 \%)$; mp $257^{\circ} \mathrm{C}-258^{\circ} \mathrm{C} .{ }^{1} \mathrm{H}$ NMR (400 MHz, DMSO-d 6 )_: 3.78 (s, 3H), 6.88 (d, J = 2.0 Hz, 1H), 6.94 (dd, J = 2.4, $8.8 \mathrm{~Hz}, 1 \mathrm{H}), 6.98$ (d, J = 8.8 Hz, 2H), 7.49 (d, J = 8.8 Hz, $2 \mathrm{H}$ ), $6.97(\mathrm{~d}, \mathrm{~J}=8.8 \mathrm{~Hz}, 1 \mathrm{H}), 8.31(\mathrm{~s}, 1 \mathrm{H}) .{ }^{13} \mathrm{C}$ NMR $\left(100 \mathrm{MHz}, \mathrm{DMSO}-\mathrm{d}_{6}\right)_{-}=55.1,102.0,113.6,114.1,115.0$, 116.6, 123.1, 124.1, 127.2, 130.0, 153.0, 157.4, 158.9, 162.3, 174.6. LC-MS: m/z: 267 [M-1]-. Anal.calcd. for $\mathrm{C}_{16} \mathrm{H}_{12} \mathrm{O}_{4}$ : C 71.64, H 4.51; found: C 71.60, H 4.55.

\subsection{7-Hydroxy-3-(4-Hydroxyphenyl)-4H-Chromen-4-One (9d) (Table 2, Entry 4)}

Pale brown powder; yield (method A) $670 \mathrm{mg}$ (88\%); mp $310^{\circ} \mathrm{C}-312^{\circ} \mathrm{C} .{ }^{1} \mathrm{H}$ NMR (400 MHz, DMSO-d $)_{2}$ : 6.79 (d, J = 8.4 Hz, 2H), 6.83 (d, J = 2.0 Hz, 1H), 6.91 (dd, J = 2.0, $8.0 \mathrm{~Hz}, 1 \mathrm{H}), 7.36$ (d, J = 8.4 Hz, $2 \mathrm{H}$ ), 7.96 (d, $\mathrm{J}=8.0 \mathrm{~Hz}, 1 \mathrm{H}), 8.28(\mathrm{~s}, 1 \mathrm{H}), 9.55(\mathrm{~s}, 1 \mathrm{H}), 10.83(\mathrm{~s}, 1 \mathrm{H}) .{ }^{13} \mathrm{C}$ NMR $(100 \mathrm{MHz} \text {, DMSO-d })_{-}=102.0,114.9,115.1$, 122.5, 123.5, 127.2, 130.0, 157.2, 157.3, 162.4, 174.7. LC-MS: m/z: 253 [M-1]-. Anal.calcd. for $\mathrm{C}_{15} \mathrm{H}_{10} \mathrm{O}_{4}: \mathrm{C}$ 70.86, H 3.96; found: C 70.85, H 3.98.

\subsection{7-Hydroxy-3-(2, 4-Dimethoxyphenyl)-4H-Chromen-4-One (9e) (Table 2, Entry 5)}

Off white solid; yield (method A) $795 \mathrm{mg}(89 \%)$; mp $265^{\circ} \mathrm{C}-270^{\circ} \mathrm{C} .{ }^{1} \mathrm{H}$ NMR (400 MHz, DMSO-d 6 )_: 3.70 (s, 3H), 3.80 (s, 3H), 6.56 (d, J = 8.0 Hz, 1H), 6.63 (s, 1H), 6.86 (s, 1H), 6.93 (d, J = 8.8 Hz, 1H), 7.13 (d, J = 8.0 $\mathrm{Hz}, 1 \mathrm{H}), 7.92$ (d, J = 8.8 Hz, 1H), $8.11(\mathrm{~s}, 1 \mathrm{H}), 10.73(\mathrm{~s}, 1 \mathrm{H}){ }^{13} \mathrm{C}$ NMR $\left(100 \mathrm{MHz}, \mathrm{DMSO}-\mathrm{d}_{6}\right)_{-}=55.2,55.5$, 98.6, 102.1, 104.6, 113.5, 114.9, 116.5, 121.5, 127.1, 132.0, 153.8, 157.4, 158.4, 160.6, 162.4, 174.3. LC-MS: m/z: 297 [M-1] ${ }^{-}$. Anal. calcd. for $\mathrm{C}_{17} \mathrm{H}_{14} \mathrm{O}_{5}$ : C 68.45, H 4.73; found: C 68.46, H 4.75.

Table 2. Synthesis of isoflavones (9a-9h).

\begin{tabular}{|c|c|c|c|c|c|c|c|c|}
\hline S. No. & Entry & $\mathrm{R}_{1}$ & $\mathrm{R}_{2}$ & $\mathrm{R}_{3}$ & $\mathrm{R}_{4}$ & $\mathrm{R}_{5}$ & $\mathrm{R}_{6}$ & 9 Yield (\%) \\
\hline 1 & a & $\mathrm{H}$ & $\mathrm{OH}$ & $\mathrm{H}$ & $\mathrm{H}$ & $\mathrm{H}$ & $\mathrm{H}$ & 88 \\
\hline 2 & b & $\mathrm{H}$ & $\mathrm{OH}$ & $\mathrm{H}$ & $\mathrm{OCH}_{3}$ & $\mathrm{H}$ & $\mathrm{H}$ & 89 \\
\hline 3 & c & $\mathrm{H}$ & $\mathrm{OH}$ & $\mathrm{H}$ & $\mathrm{H}$ & $\mathrm{OCH}_{3}$ & $\mathrm{H}$ & 90 \\
\hline 4 & d & $\mathrm{H}$ & $\mathrm{OH}$ & $\mathrm{H}$ & $\mathrm{H}$ & $\mathrm{OH}$ & $\mathrm{H}$ & 88 \\
\hline 5 & e & $\mathrm{H}$ & $\mathrm{OH}$ & $\mathrm{OCH}_{3}$ & $\mathrm{H}$ & $\mathrm{OCH}_{3}$ & $\mathrm{H}$ & 89 \\
\hline 6 & $\mathrm{f}$ & $\mathrm{OH}$ & $\mathrm{OH}$ & $\mathrm{H}$ & $\mathrm{H}$ & $\mathrm{H}$ & $\mathrm{H}$ & $75,89^{b}$ \\
\hline 7 & g & $\mathrm{OH}$ & $\mathrm{OH}$ & $\mathrm{H}$ & $\mathrm{OCH}_{3}$ & $\mathrm{H}$ & $\mathrm{H}$ & $70,85^{b}$ \\
\hline 8 & $\mathrm{~h}$ & $\mathrm{OH}$ & $\mathrm{OH}$ & $\mathrm{H}$ & $\mathrm{H}$ & $\mathrm{OCH}_{3}$ & $\mathrm{H}$ & $75,90^{\mathrm{b}}$ \\
\hline
\end{tabular}

${ }^{\mathrm{a}}$ Unoptimized condition; ${ }^{\mathrm{b}}$ Deoxybenzoins were isolated and converted into isoflavones. 


\subsection{7,8-Dihydroxy-3-Phenyl-4H-Chromen-4-One (9f) (Table 2, Entry 6)}

Pale brown solid; yield (method A) $571 \mathrm{mg}$ (75\%), yield (method B) $677 \mathrm{mg}(89 \%)$; mp $200^{\circ} \mathrm{C}-205^{\circ} \mathrm{C} .{ }^{1} \mathrm{H}$ NMR (400 MHz, DMSO-d $)_{6}$ : 6.98 (d, J = 8.8 Hz, 1H), 7.37 - 7.44 (m, 3H), 7.49 (d, J = 8.8 Hz, 1H), 7.59-7.57 $(\mathrm{m}, 2 \mathrm{H}), 8.43(\mathrm{~s}, 1 \mathrm{H}), 9.46(\mathrm{~s}, 1 \mathrm{H}), 10.33(\mathrm{~s}, 1 \mathrm{H}) .{ }^{13} \mathrm{C}$ NMR $\left(100 \mathrm{MHz}, \mathrm{DMSO}-\mathrm{d}_{6}\right) \_=114.2,115.6,117.4,123.0$, 127.5, 128.0, 128.9, 132.2, 132.9, 146.7, 150.1, 153.5, 174.8. LC-MS: m/z: 253 [M-1] . Anal.calcd. for $\mathrm{C}_{15} \mathrm{H}_{10} \mathrm{O}_{4}$ : C 70.86, $\mathrm{H}$ 3.96; found: C 70.84, $\mathrm{H} 4.00$.

\subsection{7,8-Dihydroxy-3-(3-Methoxyphenyl)-4H-Chromen-4-One (9g) (Table 2, Entry 7)}

Brown solid; yield (method A) $597 \mathrm{mg}$ (70\%), yield (method B) $725 \mathrm{mg}$ (85\%); mp $216^{\circ} \mathrm{C}-218^{\circ} \mathrm{C} .{ }^{1} \mathrm{H} \mathrm{NMR}$ (400 MHz, DMSO-d $)_{6}$ : 3.78 (s, 3H), 6.94 (d, J = 7.6 Hz, 1H), 6.97 (d, J = 8.8 Hz, 1H), 7.14 - 7.17 (m, $\left.2 \mathrm{H}\right)$, 7.33 (t, J = $7.6 \mathrm{~Hz}, 1 \mathrm{H}), 7.49$ (d, J = $8.8 \mathrm{~Hz}, 1 \mathrm{H}), 8.44$ (s, $1 \mathrm{H}) 9.41$ (s, 1H), 10.32 (s, $1 \mathrm{H}) .{ }^{13} \mathrm{C} \mathrm{NMR}(100 \mathrm{MHz}$ DMSO-d $)_{-}=55.0,113.1,114.2,114.7$ 115.7, 117.4, 121.2, 122.7, 129.0, 132.9, 133.5, 146.6, 150.1, 153.6, 158.9, 174.7. LC-MS: m/z: 283 [M-1] . Anal. calcd. for $\mathrm{C}_{16} \mathrm{H}_{12} \mathrm{O}_{5}$ : C 67.60, H 4.25; found: C 67.58, H 4.28.

\subsection{7,8-Dihydroxy-3-(4-Methoxyphenyl)-4H-Chromen-4-One (9h) (Table 2, Entry 8)}

Pale Brown solid; yield (method A) $640 \mathrm{mg}$ (75\%), yield (method B) $768 \mathrm{mg}(90 \%)$; mp $252^{\circ} \mathrm{C}-254^{\circ} \mathrm{C}$. ${ }^{1} \mathrm{H}$ NMR (400 MHz, DMSO-d $)_{6}$ : 3.79 (s, 3H), 6.96 (d, J = 8.8 Hz, 1H), 6.99 (d, J = 8.8 Hz, 2H), 7.48 (d, J = 8.8 $\mathrm{Hz}, 1 \mathrm{H}), 7.52$ (d, J = 8.8 Hz, 2H), 8.38 (s, 1H), 9.42 (s, 1H), 10.29 (s, 1H). ${ }^{13} \mathrm{C}$ NMR $\left(100 \mathrm{MHz}, \mathrm{DMSO}_{6}\right)_{-}=$ 55.1, 113.5, 114.1, 115.6, 117.4, 122.6, 124.4, 130.0, 132.8, 146.7, 149.8, 150.0, 152.8, 158.9, 175.0. LC-MS: m/z: 283 [M-1] ${ }^{-}$. Anal. calcd. for $\mathrm{C}_{16} \mathrm{H}_{12} \mathrm{O}_{5}$ : C 67.60, H 4.25; found: C 67.59, H 4.28.

\section{General Experimental Procedure for Homo-Isoflavones (10a-10f)}

\subsection{Method A}

A mixture of substituted phenol (3 mmol), 3-phenylpropanoic acid ( $3 \mathrm{mmol})$, and $\mathrm{BF}_{3} \cdot \mathrm{Et}_{2} \mathrm{O}$ (15 mmol) was refluxed at $90^{\circ} \mathrm{C}$ for 90 min under Nitrogen atmosphere. The reaction mixture was then cooled to $10^{\circ} \mathrm{C}$ and $\mathrm{DMF}$ (4.6 mL) was added drop wise. The above reaction mixture was then added drop wise with stirring into vilsmeier reagent $(4.5 \mathrm{mmol})$ at room temperature. After completion of addition, the reaction mixture was stirred at $60^{\circ} \mathrm{C}$ for $30-40 \mathrm{~min}$ and poured into boiling dilute $\mathrm{HCl}$ slowly and cooled. The solution was extracted with ethylacetate $(30 \mathrm{~mL} \times 2)$ and the organic layer was dried over anhydrous $\mathrm{Na}_{2} \mathrm{SO}_{4}$. The crude obtained after evaporation of the solvent was chromatographed over a silica gel column using chloroform-methanol mixtures as eluent to give homo-isoflavones (10a-10h).

\subsection{Method B}

A mixture of substituted phenol ( $3 \mathrm{mmol}$ ), 3-phenylpropanoic acid ( $3 \mathrm{mmol})$, and $\mathrm{BF}_{3} \cdot \mathrm{Et}_{2} \mathrm{O}(9 \mathrm{mmol})$ was refluxed at $90^{\circ} \mathrm{C}$ for 90 min under Nitrogen atmosphere. The mixture was then poured into NaOAc solution (100 $\mathrm{mL}, 10 \%)$ and allowed to stand for $4 \mathrm{hr}$ and the solution was extracted with EtOAc $(3 \times 100 \mathrm{~mL})$. The combined organic layer was washed with water $(20 \mathrm{~mL})$ and brine $(20 \mathrm{~mL})$ and dried over anhydrous $\mathrm{Na}_{2} \mathrm{SO}_{4}$. The crude obtained after evaporation of the solvent was chromatographed over a silica gel column using hexane-EtOAc mixtures as eluent to give dihydrchalcones (8d-8f). The purified materials were then used for the synthesis of homoisoflavones. A mixture of dihydrochalcone $(3 \mathrm{mmol})$ and $\mathrm{BF}_{3} \cdot \mathrm{Et}_{2} \mathrm{O}(7.5 \mathrm{mmol})$ was cooled to $10^{\circ} \mathrm{C}$ and DMF (4.6 mL) was added drop wise. The cyclization procedure and work up are similar to method A.

\subsection{3-Benzyl-7-Hydroxy-4H-Chromen-4-One (10a) (Table 3, Entry 1)}

Pale pink solid; yield (method A) $635 \mathrm{mg}$ (84\%); mp $210^{\circ} \mathrm{C}-214^{\circ} \mathrm{C} .{ }^{1} \mathrm{H}$ NMR (400 MHz, DMSO-d 6 )_: 3.67 (s, 2H), 6.82 (d, J = 2.4 Hz, 1H), 6.89 (dd, J = 2.4, $8.8 \mathrm{~Hz}, 1 \mathrm{H}), 7.14-7.18(1 \mathrm{H}), 7.23-7.29$ (m, $4 \mathrm{H}), 7.87(\mathrm{~d}, \mathrm{~J}=$ $8.8 \mathrm{~Hz}, 1 \mathrm{H}), 8.17$ (s, 1H), $10.72(\mathrm{~s}, 1 \mathrm{H}) .{ }^{13} \mathrm{C}$ NMR $\left(100 \mathrm{MHz}, \mathrm{DMSO}-\mathrm{d}_{6}\right)=30.6,102.1,114.9,116.2,122.8$, 125.9, 126.7, 128.1, 128.4, 139.7, 153.2, 157.7, 162.4, 175.4. LC-MS: m/z: 251 [M-1]. Anal. calcd. for $\mathrm{C}_{16} \mathrm{H}_{12} \mathrm{O}_{3}$ : C 76.18, $\mathrm{H}$ 4.79; found: C 76.14, $\mathrm{H} 4.82$. 
Table 3. Synthesis of homoisoflavones (10a-10f).

\begin{tabular}{ccccccccc}
\hline S. No. & Entry & $\mathrm{R}_{1}$ & $\mathrm{R}_{2}$ & $\mathrm{R}_{3}$ & $\mathrm{R}_{4}$ & $\mathrm{R}_{5}$ & $\mathrm{R}_{6}$ & 10 Yield (\%) \\
\hline 1 & $\mathrm{a}$ & $\mathrm{H}$ & $\mathrm{OH}$ & $\mathrm{H}$ & $\mathrm{H}$ & $\mathrm{H}$ & $\mathrm{H}$ & 84 \\
2 & $\mathrm{~b}$ & $\mathrm{H}$ & $\mathrm{OH}$ & $\mathrm{H}$ & $\mathrm{H}$ & $\mathrm{OCH}_{3}$ & $\mathrm{H}$ & 87 \\
3 & $\mathrm{c}$ & $\mathrm{H}$ & $\mathrm{OH}$ & $\mathrm{H}$ & $\mathrm{H}$ & $\mathrm{OH}$ & $\mathrm{H}$ & 89 \\
4 & $\mathrm{~d}$ & $\mathrm{H}$ & $\mathrm{OH}$ & $\mathrm{OCH}_{3}$ & $\mathrm{H}$ & $\mathrm{H}$ & $\mathrm{OCH}_{3}$ & 88 \\
5 & $\mathrm{e}$ & $\mathrm{OH}$ & $\mathrm{OH}$ & $\mathrm{H}$ & $\mathrm{H}$ & $\mathrm{OCH}_{3}$ & $\mathrm{H}$ & $78,88^{\mathrm{b}}$ \\
6 & $\mathrm{f}$ & $\mathrm{OH}$ & $\mathrm{OH}$ & $\mathrm{OCH}_{3}$ & $\mathrm{H}$ & $\mathrm{H}$ & $\mathrm{OCH}_{3}$ & $80,85^{\mathrm{b}}$ \\
\hline
\end{tabular}

${ }^{\mathrm{a} U n o p t i m i z e d ~ c o n d i t i o n . ~}{ }^{\mathrm{b}}$ Dihydrochalcones were isolated and converted into homoisoflavones.

\subsection{7-Hydroxy-3-(4-Methoxybenzyl)-4H-Chromen-4-One (10b) (Table 3, Entry2)}

Pale brown solid; yield (method A) $736 \mathrm{mg}(87 \%)$; mp $161{ }^{\circ} \mathrm{C}-165^{\circ} \mathrm{C} .{ }^{1} \mathrm{H}$ NMR (400 MHz, DMSO-d 6 )_: 3.59 (s, 2H), 3.69 (s, 3H), 6.81 - 6.83 (m, 3H), 6.89 (dd, J = 2.0, 8.8 Hz, 1H), 7.20 (d, J = 8.4 Hz, $2 \mathrm{H}), 7.87$ (d, J = 8.8 $\mathrm{Hz}, 1 \mathrm{H}), 8.12$ (s, $1 \mathrm{H}), 10.71$ (s, 1H). ${ }^{13} \mathrm{C}$ NMR (100 MHz, DMSO-d $)_{-}=29.8,54.9,102.1,113.6,114.9,116.2$, 123.3, 126.7, 129.0, 129.5, 131.5, 153.0, 157.6, 157.7, 162.4, 175.4. LC-MS: m/z: 281 [M-1]-. Anal.calcd. for $\mathrm{C}_{17} \mathrm{H}_{14} \mathrm{O}_{4}$ : C 72.33, $\mathrm{H}$ 5.00; found: C 72.30, H 5.05.

\subsection{7-Hydroxy-3-(4-Hydroxybenzyl)-4H-Chromen-4-One (10c) (Table 3, Entry3)}

Colorless solid; yield (method A) $715 \mathrm{mg}$ (89\%); mp 210 ${ }^{\circ} \mathrm{C}-212^{\circ} \mathrm{C} .{ }^{1} \mathrm{H}$ NMR (400 MHz, DMSO-d 6 )_: 3.52 (s, 2H), 6.64 (d, J = 8.4 Hz, 2H), 6.79 (d, J = 2.0 Hz, 1H), 6.89 (dd, J = 8.8, 2.0 Hz, 1H), 7.07 (d, J = 8.4 Hz, 2H), 7.86 (d, J = 8.8 Hz, 1H), 8.05 (s, 1H), 9.85 (s, 1H), 10.75 (s, 1H) ${ }^{13} \mathrm{C}$ NMR (100 MHz, DMSO-d $)_{-}=30.2,102.1$, 114.5, 115.1, 116.3, 123.9, 126.7, 128.9, 129.4, 152.3, 155.5, 157.8, 162.3, 176.3. LC-MS: m/z: 267 [M-1]. Anal.calcd. for $\mathrm{C}_{16} \mathrm{H}_{12} \mathrm{O}_{4}$ : C 71.64, $\mathrm{H}$ 4.51; found: C 71.62, H 4.54.

\subsection{7-Hydroxy-3-(2, 5-Dimethoxybenzyl)-4H-Chromen-4-One (10d) (Table 3, Entry4)}

Light brown solid; yield (method A) $823 \mathrm{mg}(88 \%)$; mp $184^{\circ} \mathrm{C}-188^{\circ} \mathrm{C} .{ }^{1} \mathrm{H}$ NMR (400 MHz, DMSO-d 6 )_: 3.59 (s, 2H), 3.69 (s, 3H), 3.75 (s, 3H), $6.70-6.75$ (m, 2H), 6.80 (d, J = 2.0 Hz, 1H), ), 6.85 (d, J = 8.8 Hz, $1 \mathrm{H}), 6.90$ (dd, J = 2.0, $8.8 \mathrm{~Hz}, 1 \mathrm{H}), 7.88(\mathrm{~s}, 1 \mathrm{H}), 7.89$ (d, J = $8.8 \mathrm{~Hz}, 1 \mathrm{H}), 10.68(\mathrm{~s}, 1 \mathrm{H}) .{ }^{13} \mathrm{C}$ NMR $(100 \mathrm{MHz}$, DMSO$\left.\mathrm{d}_{6}\right)_{-}=25.2$, 55.2, 55.7, 102.1, 111.3, 111.6, 114.9, 116.1, 116.4, 121.7, 126.7, 128.0, 151.1, 151.2, 152.9, 153.2, 157.7, 162.4, 175.4. LC-MS: m/z: 311 [M-1] . Anal.calcd. for $\mathrm{C}_{18} \mathrm{H}_{16} \mathrm{O}_{5}$ : C 69.22, H 5.16; found: C 69.19, H 5.19 .

\subsection{7,8-Dihydroxy-3-(4-Methoxybenzyl)-4H-Chromen-4-One (10e) (Table 3, Entry 5)}

Colorless solid; yield (method A) $698 \mathrm{mg}$ (78\%), yield (method B) $785 \mathrm{mg}(88 \%)$; mp $250^{\circ} \mathrm{C}-253^{\circ} \mathrm{C} .{ }^{1} \mathrm{H} \mathrm{NMR}$

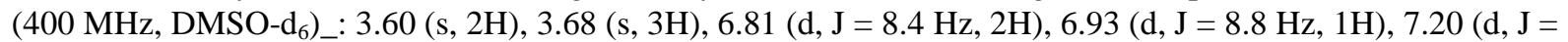
$8.4 \mathrm{~Hz}, 2 \mathrm{H}), 7.39(\mathrm{~d}, \mathrm{~J}=8.8 \mathrm{~Hz}, 1 \mathrm{H}), 8.19(\mathrm{~s}, 1 \mathrm{H}), 9.34(\mathrm{~s}, 1 \mathrm{H}), 10.20(\mathrm{~s}, 1 \mathrm{H}) .{ }^{13} \mathrm{C}$ NMR $\left(100 \mathrm{MHz}, \mathrm{DMSO}_{-}\right)_{-}$ = 29.8, 54.9, 113.6, 114.0, 115.1, 117.1, 122.7, 129.4, 131.6, 132.8, 147.0, 149.8, 152.8, 157.6, 175.9. LC-MS: m/z: 297 [M-1] ${ }^{-}$. Anal.calcd. for $\mathrm{C}_{17} \mathrm{H}_{14} \mathrm{O}_{5}$ : C 68.45, H 4.73; found: C 68.42, H 4.79.

\subsection{7,8-Dihydroxy-3-(2, 5-Dimethoxybenzyl)-4H-Chromen-4-One (10f)} (Table 3, Entry 6)

Pale brown solid; yield (method A) $786 \mathrm{mg}$ (80\%), yield (method B) $836 \mathrm{mg}(85 \%)$; mp $226^{\circ} \mathrm{C}-230^{\circ} \mathrm{C} .{ }^{1} \mathrm{H}$ NMR (400 MHz, DMSO-d ) $_{-}: 3.60$ (s, 2H), 3.64 (s, 3H), 3.75 (s, 3H), 6.72 - 6.75 (m, 2H), 6.88 (d, J = 8.4 Hz, 1H), 6.93 (d, J = 8.8 Hz, 1H), 7.39 (d, J = $8.8 \mathrm{~Hz}, 1 \mathrm{H}), 7.98(\mathrm{~s}, 1 \mathrm{H}), 9.32$ (s, 1H), 10.20 (s, $1 \mathrm{H}) .{ }^{13} \mathrm{C} \mathrm{NMR}(100$ MHz, DMSO-d $)_{6}=25.2$, 55.2, 55.8, 111.3, 111.5, 114.0, 115.1, 116.4, 116.9, 121.1, 128.1, 132.8, 147.0, 149.8, 151.2, 152.9, 153.0, 175.9. LC-MS: m/z: 327 [M-1] ${ }^{-}$. Anal. calcd. For $\mathrm{C}_{18} \mathrm{H}_{16} \mathrm{O}_{6}$ : C 65.85, H 4.91; found: C 65.81, H 4.96 . 


\section{Acknowledgements}

The author is grateful to the Department of Organic Chemistry \& FDW, Andhra University, Visakhapatnam, India for giving him the opportunity to pursue his $\mathrm{PhD}$.

\section{References}

[1] McClure, J.W., Harborne, J.B., Mabry, T.J. and Mabry, H. (1975) The Flavonoids. Chapman and Hall, London, 970.

[2] Attassi, G., Briet, P., Berthelon, J.P. and Collonges, F. (1985) Synthesis and Antitumor Activity of Some (8-Substituted 4-oxo-4H-1-benzopyrans). Journal of Medicinal Chemistry, 20, 393-402.

[3] Middleton Jr., E., Kandaswami, C. and Arborne, J.B. (1994) The Flavonoids Advances in Research Since 1986. Chapman and Hall, London, 619.

[4] Bruneton, J. (1995) Pharmacognosy, Phytochemistry and Medicinal Plants, English Translation by Hatton, C. K., Lavoisier Publishing, Paris, 265.

[5] Harborne, J.B. and Williams, C.A. (2000) Advances in Flavonoid Research Since 1992. Phytochemistry, 55, $481-504$. http://dx.doi.org/10.1016/S0031-9422(00)00235-1

[6] Miksicek, R.J. (1995) Estrogenic Flavonoids: Structural Requirements for Biological Activity. Proceedings of the Society for Experimental Biology and Medicine, 208, 44-50. http://dx.doi.org/10.3181/00379727-208-43830

[7] Peterson, G. and Branes, S. (1991) Genistein Inhibition of the Growth of Human Breast Cancer Cells: Independence from Estrogen Receptors and the Multi-Drug Resistance Gene. Biochemical and Biophysical Research Communications, 179, 661-667. http://dx.doi.org/10.1016/0006-291X(91)91423-A

[8] Bandyukova, V.A., Cherevatye, V.S., Ozimina, I.I., Andreeva, O.A., Lebedava, A.L., Davydov, V.S., Vashchenko, T. N. Postnikova, N.V., (1987) Antibacterial Activity of Flavonoids of Some Flowering Plant Species. Rastitel'nye Resursy, 23, 607; Chemical Abstract, 108, 1990, 71937v.

[9] El-Gammal, A.A. and Mansour, R.M., (1986) Antimicrobial Activities of Some Flavonoid Compounds. Zentralblatt für Mikrobiologie, 141, 561-565; Chemical Abstract, 106, 1987, 135070a.

[10] Takai, M., Yamaguchi, H., Saitoh, T. and Shibata, S. (1972) Chemical and Pharmaceutical Bulletin, 20, $2488-2490$. http://dx.doi.org/10.1248/cpb.20.2488

[11] Akiyama, T., Ishida, J., Nakagawa, S., Ogawara, H., Watanabe, S., Itoh, N., Shihuya, M. and Fukami, Y. (1987) Genistein, a Specific Inhibitor of Tyrosine-Specific Protein Kinases. Journal of Biological Chemistry, 262, 5592-5595.

[12] Baker, W., Chadderton, J., Harborne, J.B. and Ollis, W.D. (1953) A New Synthesis of Isoflavones. Part II. 5: 7: 2’Trihydroxyisoflavone. Journal of the Chemical Society, 1860-1864. http://dx.doi.org/10.1039/jr9530001860

[13] Sathe, V.R. and Venkataraman, K. (1949) A New Reaction for the Synthesis of Chromones and Isoflavones. Current Science, 18, 373; Chemical Abstract, 44, 1950, 8916.

[14] Wahala, K. and Hase, T.A. (1991) Expedient Synthesis of Polyhydroxyisoflavones. Journal of the Chemical Society, Perkin Transactions 1, 3005-3008. http://dx.doi.org/10.1039/p19910003005

[15] Balasubramanian, S., Ward, D.L. and Nair, M.G. (2000) The First Isolation and Crystal Structure of a Boron Difluoro Complex (Isoflavone Yellow). Biologically Active Intermediates Produced during Isoflavone Synthesis. Journal of the Chemical Society, Perkin Transactions 1, 567-569. http://dx.doi.org/10.1039/a908915b

[16] Kagal, S.A., Nair, P.M. and Venkataraman, K. (1962) A Synthesis of Isoflavones by a Modified Vilsmeier-Haack Reaction. Tetrahedron Letters, 3, 593.

[17] Wessely, F., Kornfeld, L. and Lechner, F. (1953) Über die Synthese von Daidzein und von 7-Oxy-4'-Methoxy-Isoflavon. Berichte der deutschen chemischen Gesellschaft (A and B Series), 66, 685-687. http://dx.doi.org/10.1002/cber.19330660515

[18] Bognar, R. and Levai, A. (1973) Synthesis of 4- $\beta$-Glucosyloxydeoxybenzoins and Their Conversion into 7- $\beta$-Glucosyloxyisoflavones. Acta Chimica Academiae Scientiarum Hungaricae, 77, 435.

[19] Levai, A. and Bognar, R. (1974) Preparation of Deoxybenzoin Glycosides and Their Conversion into Isoflavone Glycosides. Kemiai Kozlemenyek, 41, 17-25.

[20] Liu, D.F. and Cheng, C.C. (1991) A Facile and Practical Preparation of 5,7-Dihydroxy-3-(4-nitrophenyl)-4H-1-benzopyran-4-one. Journal of Heterocyclic Chemistry, 28, 1641-1642. http://dx.doi.org/10.1002/jhet.5570280632

[21] Krishnamurty, H.G. and Prasad, J.S. (1977) A New Synthesis of Isoflavones Using “Active Formate”. Tetrahedron Letters, 18, 3071-3072. http://dx.doi.org/10.1016/S0040-4039(01)83160-9

[22] Miki, Y., Fujita, R. and Matsushita, K.I. (1998) Oxidative Rearrangement of Pentaalkoxychalcones with Phenyliodine(III) Bis(trifluoroacetate) (PIFA): Synthesis of $( \pm$ )-10-Bromopterocarpin and ( \pm )-Pterocarpin. Journal of the Che- 
mical Society, Perkin Transactions 1, 2533-2536. http://dx.doi.org/10.1039/a803561j

[23] Kawamura, Y., Maruyama, M., Tokuoka, T. and Tsukayama, M. (2000) Synthesis of Isoflavones from 2'-Hydroxychalcones Using Poly[4-(diacetoxy)iodo]styrene or Related Hypervalent Iodine Reagent. Synthesis, 2490-2496.

[24] Faskas, L., Gottsegen, A., Nogradi, M. and Antus, S. (1972) Direct Conversion of 2'-Hydroxychalcones into Isoflavones Using Thallium(III) Nitrate: Synthesis of $( \pm)$-Sophorol and ( \pm )-Mucronulatol. Journal of the Chemical Society, Chemical Communications, 825-826. http://dx.doi.org/10.1039/c39720000825

[25] Farkas, L., Gottsegen, A., Nogradi, M. and Antus, S. (1974) Synthesis of Sophorol, Violanone, Lonchocarpan, Claussequinone, Philenopteran, Leiocalycin, and Some Other Natural Isoflavonoids by the Oxidative Rearrangement of Chalcones with Thallium(III) Nitrate. Journal of the Chemical Society, Perkin Transactions 1, 305-312. http://dx.doi.org/10.1039/p19740000305

[26] Farkas, L., Antus, S. and Nogradi, M. (1974) The Oxidative Rearrangement of Chalcones by Thallium(III) Nitrate, II. New Synthesis of Flemichapparin-B and Flemichapparin-C. Acta Chimica (Academiae Scientiarum) Hungaricae, 82, 225-230.

[27] Antus, S., Farkas, L., Kardos-Balogh, Z. and Nógrádi, M. (1975) Oxidative Umlagerung von Chalkonen mit Thallium(III)nitrat, IV. Synthese des Dalpatins, Fujikinins, Glyciteins und anderer natürlicher Isoflavone. Chemische Berichte, 108, 3883-3893. http://dx.doi.org/10.1002/cber.19751081221

[28] Antus, S., Farkas, L., Gottsegen, A., Kardos-Balogh, Z. and Nogradi, M. (1976) Oxidative Umlagerung von Chalconen mit Thallium(III)-nitrat, VI Synthese der Isoflavonoide Jamaicin und Leiocarpin. Chemische Berichte, 109, 3811-3816. http://dx.doi.org/10.1002/cber.19761091208

[29] Antus, S., Boross, F. and Nógrádi, M. (1977) Alkali-Catalysed Alkoxy Exchange, Alcohol Elimination, and Hydrolysis of Acetals Having a Dissociable $\alpha$-Proton. Journal of the Chemical Society, Chemical Communications, 333-334. http://dx.doi.org/10.1039/c39770000333

[30] Ollis, W.D., Ormand, K.L. and Sutherland, I.O. (1968) The Oxidative Rearrangement of Chalcones by Thallic Acetate: A Chemical Analogy for Isoflavone Biosynthesis. Journal of the Chemical Society, Chemical Communications, 12371238.

[31] Ollis, W.D., Ormand, K.L. and Sutherland, I.O. (1970) The Oxidative Rearrangement of Olefins by Thallium(III) Acetate. Part I. Oxidative Rearrangement of Chalcones. Journal of the Chemical Society C, 119-124.

[32] Ollis, W.D., Ormand, K.L., Roberts, R.J. and Sutherland, I.O. (1979) The Oxidative Rearrangement of Olefins by Thallium(III) Acetate. Part II. Synthesis of Isoflavones. Journal of the Chemical Society C, 125-128.

[33] Grover, S.K., Jain, A.C. and Seshadri, T.R. (1963) A Convenient Synthesis of Isoflavones and Di-O-Methylangolensin Using Aryl Migration. Indian Journal of Chemistry, 1, 517.

[34] Jain, A.C., Lal, P. and Seshadri, T.R. (1969) Synthesis of 3'-Hydroxyformononetin. Indian Journal of Chemistry, 7, 305.

[35] Kinoshita, T., Ichinose, K. and Sankawa, U. (1990) One-Step Conversion of Flavanones into Isoflavones: A New Facile Biomimetic Synthesis of Isoflavones. Tetrahedron Letters, 31, 7355-7356. http://dx.doi.org/10.1016/S0040-4039(00)88565-2

[36] Weber-Schilling, C.A. and Wanzlick, H.W. (1971) Synthese von 2'.4'.5'-trihydroxylierten Isoflavonen. Chemische Berichte, 104, 1518-1523. http://dx.doi.org/10.1002/cber.19711040520

[37] Crombie, L., Freeman, P.W. and Whiting, D.A. (1973) A New Synthesis of Rotenoids. Application to 9-Demethylmunduserone, Mundeserone, Rotenonic Acid, Dalpanol, and Rotenone. Journal of the Chemical Society, Perkin Transactions 1, 1277-1285. http://dx.doi.org/10.1039/p19730001277

[38] Yokoe, I., Sugita, Y. and Shirataki, Y. (1989) Facile Synthesis of Isoflavones by the Cross-Coupling Reaction of 3-Iodochromone with Arylboronic Acids. Chemical and Pharmaceutical Bulletin, 37, 529-530.

[39] Qualig, M.G., Desideri, N., Bossu, E., Sgro, R. and Conti, C. (1999) Enantioseparation and Anti-Rhinovirus Activity of 3-Benzylchroman-4-Ones. Chirality, 11, 495-500. http://dx.doi.org/10.1002/(SICI)1520-636X(1999)11:5/6<495::AID-CHIR23>3.0.CO;2-5

[40] Desideri, N., Olivieri, S., Stein, M.L., Sgro, R., Orsi, N. and Conti, C. (1997) Synthesis and Anti-Picornavirus Activity of Homo-Isoflavonoids. Antiviral Chemistry and Chemotherapy, 8, 545-555.

[41] Siddaiah, V., Rao, V.C., Venkateswarlu, S., Krishnaraju, A.V. and Subbaraju, G.V. (2006) Synthesis, Stereochemical Assignments, and Biological Activities of Homoisoflavonoids. Bioorganic \& Medicinal Chemistry, 14, 2545-2551. http://dx.doi.org/10.1016/j.bmc.2005.11.031

[42] Malhotra, S., Sharma, V.K. and Parmar, V.S. (1988) Synthesis of Three New Dihydropyranochalcones: Structural Revision of Crotmadine, an Antifungal Constituent of Crotalarla Madurensis. Journal of Natural Products, 51, 578-581.

[43] Farkas, L., Gottsegen, Á., Nógrádi, M. and Strelisky, J. (1971) Synthesis of Homoisoflavanones-II: Constituents of Eu- 
comis autumn alis and E. Punctata. Tetrahedron, 27, 5049-5054.

[44] Bass, R.J. (1976) Synthesis of Chromones by Cyclization of 2-Hydroxyphenyl Ketones with Boron Trifluoride-Diethyl Ether and Methanesulphonyl Chloride. Journal of the Chemical Society, Chemical Communications, 78-79. http://dx.doi.org/10.1039/c39760000078

[45] Siddaiah, V., Rao, C.V., Venkateswarlu, S. and Subbaraju, G.V. (2006) A Concise Synthesis of Polyhydroxydihydrochalcones and Homoisoflavonoids. Tetrahedron, 62, 841-846.

[46] Kimura, Y., Matsuura, D., Hanawa, T. and Kobayashi, Y. (2012) New Preparation Method for Vilsmeier Reagent and Related Imidoyl Chlorides. Tetrahedron Letters, 53, 1116-1118. http://dx.doi.org/10.1016/j.tetlet.2011.12.087

[47] Vilsmeier, A. and Haack, A. (1927) Über die Einwirkung von Halogenphosphor auf Alkyl-formanilide. Eine neue Methode zur Darstellung sekundärer und tertiärer p-Alkylamino-benzaldehyde. Berichte der deutschen chemischen Gesellschaft, Serie B, 60, 119-122. http://dx.doi.org/10.1002/cber.19270600118

[48] Marson, C.M. (1992) Reactions of Carbonyl Compounds with (Monohalo) Methyleniminium Salts (Vilsmeier Reagents). Tetrahedron, 48, 3659-3726. http://dx.doi.org/10.1016/S0040-4020(01)92263-X

[49] Panasenko, A., Polyanska, N.L. and Starkov, S.P. (1994) Zhurnal Obshchei Khimii, 64, 673-676.

[50] Pelter, A. and Foot, S. (1976) A New Convenient Synthesis of Isoflavones. Synthesis, 326.

[51] Yoder, L., Cheng, E. and Burroughs, W. (1954) Synthesis of Estrogenic Isoflavone Derivatives. Journal of the Iowa Academy of Science, 61, 271-276. 
Scientific Research Publishing (SCIRP) is one of the largest Open Access journal publishers. It is currently publishing more than 200 open access, online, peer-reviewed journals covering a wide range of academic disciplines. SCIRP serves the worldwide academic communities and contributes to the progress and application of science with its publication.

Other selected journals from SCIRP are listed as below. Submit your manuscript to us via either submit@scirp.org or Online Submission Portal.
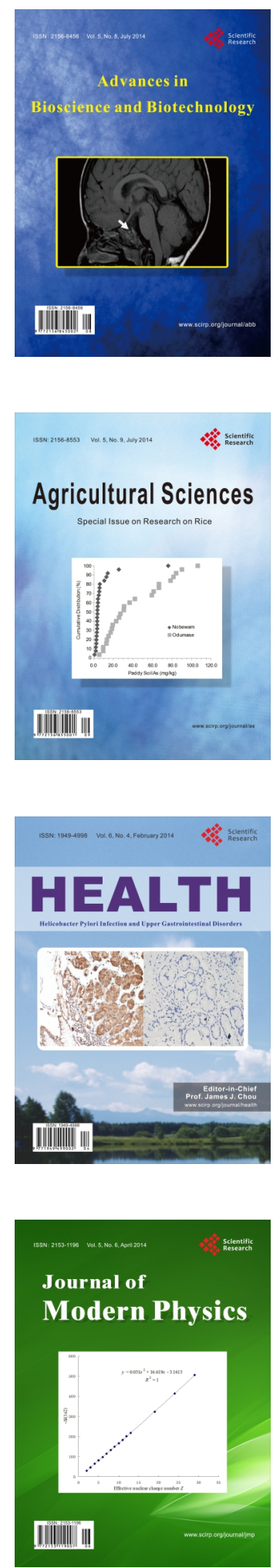
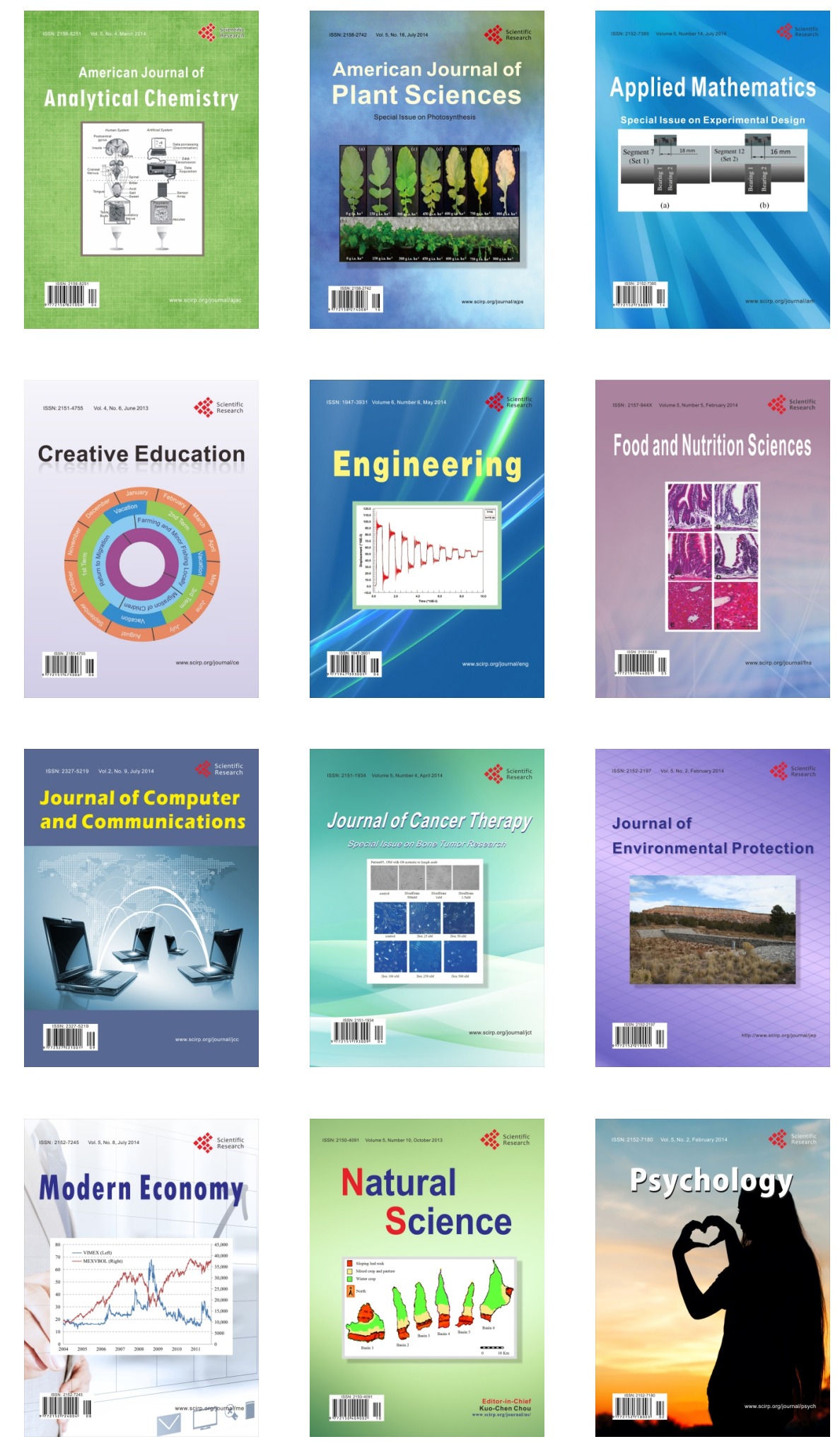